\title{
Second-harmonic generation in absorptive media
}

\author{
Gilad Almogy and Ammon Yariv \\ California Institute of Technology, Pasadena, California 91125
}

Received August 17, 1994

\begin{abstract}
The solution of the coupled-wave equations for second-harmonic generation in a near-resonant three-level system is extended to include absorption. It is shown, within second-order perturbation theory, that double resonance is the optimal conversion condition, despite absorption enhancement. We extend the solution numerically, using nonperturbative susceptibilities derived within the rotating-wave approximation, to saturating intensities and discuss the modifications to the perturbative conclusions as well as the regimes of validity for the various approximations.
\end{abstract}

The propagation equations for second-harmonic generation (SHG) are typically ${ }^{1}$ presented for transparent materials with nondispersive SHG coefficients. Because any nonlinear-optical material may be viewed as a summation of discrete level systems, the transparency assumption is a priori unjustified. Whereas all optical transitions contribute to the absorption, only the asymmetrical ones contribute to SHG. Furthermore, in a three-level system the SHG is inversely proportional to the product of the detuning of the first and the second harmonics, whereas the absorption of each harmonic is inversely proportional to the respective detuning squared. Hence the ratio of the SHG to the dominating absorption is, at best, unchanged with detuning from resonance. Recently, ${ }^{2-6}$ following the advent of quantum-well systems with tailored intersubband transition (ISBT) energies, there has been heightened interest in resonant nonlinearities. Several calculations and assumptions have been made ${ }^{5-8}$ regarding the optimal detunings and the maximal attainable conversion efficiency, but the conclusions vary. We use SHG coefficients derived from second-order perturbation theory to solve the coupled-wave equations in the nondepleted approximation. The results show that double resonance is the optimal conversion condition but that near-resonance saturation limits the use of second-order perturbation theory to small conversion efficiencies. This justifies the neglect of depletion of the first harmonic in the perturbative regime but calls for a nonperturbative solution that extends the treatment to higher intensities. For large detunings, on the other hand, saturation is avoided, the conversion distance decreases with increasing intensity, and the absorption may be neglected. The approximate solution is then extended numerically ${ }^{5-7}$ to include the effects of depletion, dispersion, absorption, and saturation. The modifications of the perturbative nondepleted conclusions are examined, and it is shown that some detuning is preferable, in some circumstances, at high intensities but that high conversion efficiencies are obtainable on resonance, despite saturation.

To examine SHG in a three-level system, in which the first harmonic is close to the ground-to-firststate $\left(\omega_{01}\right)$ and first-to-second-state $\left(\omega_{12}\right)$ transition frequencies and the second harmonic is close to the ground-to-second-state transition frequency $\left(\omega_{02}\right)$, we write the relevant resonantly enhanced first- and second-order susceptibilities as ${ }^{1}$

$$
\begin{aligned}
\chi_{(\omega, \omega)}^{(1)} & =\frac{N \mu_{10}^{2} T_{10}}{\hbar \epsilon_{0}} \frac{1}{\left(\Delta \omega_{10}-i\right)}, \\
\chi_{(2 \omega, 2 \omega)}^{(1)} & =\frac{N \mu_{20}^{2} T_{20}}{\hbar \epsilon_{0}} \frac{1}{\left(\Delta \omega_{20}-i\right)}, \\
\chi_{(2 \omega, \omega, \omega)}^{(2)} & =\frac{N \mu_{10} \mu_{21} \mu_{20} T_{10} T_{20}}{2 \hbar^{2} \epsilon_{0}} \frac{1}{\left(\Delta \omega_{10}-i\right)\left(\Delta \omega_{20}-i\right)},
\end{aligned}
$$

where $\epsilon_{0}$ is the vacuum permittivity, $N$ is the initial population (assumed to be in the ground state), $\mu_{i j}$ is the optical dipole matrix element between the $i$ th and the $j$ th states, $T_{i j}$ is the corresponding dephasing time, and $\Delta \omega_{i j}$ is the corresponding normalized detuning, defined as $\Delta \omega_{i j}=\left[\omega_{i j}-(i-j) \omega\right] T_{i j}$. The respective absorption coefficients are hence

$$
\alpha_{\omega} \equiv \frac{\omega}{2 n c} \operatorname{Im}\left[\chi_{(\omega, \omega)}^{(1)}\right],
$$

and a phase-mismatch coefficient is defined as

$$
\beta_{2 \omega} \equiv(\omega / n c)\left\{n_{2 \omega}{ }^{2}-n_{\omega}{ }^{2}+\operatorname{Re}\left[\chi_{(2 \omega, 2 \omega)}^{(1)}\right]-\operatorname{Re}\left[\chi_{(\omega, \omega)}^{(1)}\right]\right\},
$$

where $n_{\omega}$ and $n_{2 \omega}$ are the refractive indices of the respective harmonics. The wave equations for fields of the fundamental $\left(E_{\omega}\right)$ and doubled $\left(E_{2 \omega}\right)$ harmonics reduce with the slowly varying amplitude approximation to

$$
\begin{aligned}
\frac{\mathrm{d} E_{2 \omega}}{\mathrm{d} z} & =-\left(\alpha_{2 \omega}+i \beta_{2 \omega}\right) E_{2 \omega}-i \frac{\omega}{n c} \chi_{(2 \omega, \omega, \omega)}^{(2)} E_{\omega}{ }^{2}, \\
\frac{\mathrm{d} E_{\omega}}{\mathrm{d} z} & =-\alpha_{\omega} E_{\omega}-i \frac{\omega}{2 n c} \chi_{(\omega, 2 \omega,-\omega)}^{(2)} E_{2 \omega} E_{\omega}{ }^{*},
\end{aligned}
$$

where we have defined the wave vector of the first harmonic as

$$
k \equiv(\omega / c)\left\{n_{\omega}{ }^{2}+\operatorname{Re}\left[\chi_{(\omega, \omega)}^{(1)}\right]\right\}^{1 / 2} \equiv \omega n / c
$$

in order to include the transition's contribution to the refractive index, and we have arbitrarily assigned a wave vector of $2 k$ to the second harmonic. 
Assuming phase matching $\left(\beta_{2 \omega}=0\right)$ and neglecting the depletion of the first harmonic [second term on the right-hand side of Eq. (3b)] we obtain

$$
\begin{aligned}
E_{2 \omega}(z)= & -i \frac{\omega}{n c} \frac{\chi_{(2 \omega, \omega, \omega)}^{(2)}}{\left(\alpha_{\omega}-2 \alpha_{\omega}\right)}\left[\exp \left(-2 \alpha_{\omega} z\right)\right. \\
& \left.-\exp \left(-\alpha_{2 \omega} z\right)\right] E_{\omega}(0)^{2} .
\end{aligned}
$$

The conversion is thus maximized at an optimal propagation length $\left(z_{\max }\right)$ of

$$
z_{\max }=\frac{1}{\alpha_{2 \omega}-2 \alpha_{\omega}} \ln \left(\frac{\alpha_{2 \omega}}{2 \alpha_{\omega}}\right),
$$

leading to a conversion efficiency maximum of

$$
\left|\frac{E_{2 \omega}\left(z_{\max }\right)}{E_{\omega}(0)}\right|=\frac{\omega}{n c} E_{\omega}(0)\left|\chi_{(2 \omega, \omega, \omega)}^{(2)}\right|\left[\frac{1}{\alpha_{2 \omega}}\left(\frac{2 \alpha_{\omega}}{\alpha_{2 \omega}}\right)^{\frac{2 \alpha_{\omega}}{2 \alpha^{2}-2 \alpha_{\omega}}}\right] \text {. }
$$

Substituting Eqs. (1) and (2) into Eqs. (6), we find that the conversion efficiency is maximized for $\alpha_{2 \omega}=2 \alpha_{\omega}$, i.e., $\operatorname{Im}\left[\chi_{(\omega, \omega)}^{(1)}\right]=\operatorname{Im}\left[\chi_{(2 \omega, 2 \omega)}^{(1)}\right]$, in which case the conversion efficiency at $z_{\max }=1 / \alpha_{2 \omega}$ becomes

$$
\left|\frac{E_{2 \omega}\left(z_{\max }\right)}{E_{\omega}(0)}\right|=\frac{1}{e} \frac{\left|\chi_{(2 \omega, \omega, \omega)}^{(2)}\right| E_{\omega}(0)}{\operatorname{Re}\left[\chi_{(2 \omega, 2 \omega)}^{(1)}\right]},
$$

which is proportional to the ratio of the nonlinear to linear susceptibilities. For equal optical dipole elements $\left(\mu_{02}=\mu_{01}\right)$, dephasing times $\left(T_{i j} \equiv T_{2} \forall i \neq\right.$ $j$ ), and detuning from the one- and two-photon resonances $\left(\Delta \omega_{20}=\Delta \omega_{10}\right)$ the conversion efficiency is simply

$$
\left|\frac{E_{2 \omega}\left(z_{\max }\right)}{E_{\omega}(0)}\right|=\frac{1}{e} \frac{\mu_{21} E_{\omega}(0)}{2 \hbar} T_{2} \equiv \frac{1}{e} \Omega_{21}(0) T_{2} \equiv \Omega_{\omega} / e,
$$

where $\Omega_{12}(z)$ is the Rabi frequency of the transition from the first to the second excited state and we have defined $\Omega_{\omega}$ as the unitless initial first-harmonic amplitude. The conversion efficiency is thus proportional to the optical dipole matrix element between the first and the second excited states $\left(\mu_{12}\right)$ and is independent of the detuning. Resonant enhancement does not therefore, in principle, affect the conversion efficiency, which is independent of the detuning, but the length required for it is proportional to the detuning squared by means of the absorption.

Equal detuning and equal dipole elements that lead to $\alpha_{2 \omega}=2 \alpha_{\omega}$ also lead to phase matching $\left(\beta_{2 \omega}=0\right.$ ) for nondispersive bulk [Eq. (2b)]. Nonequal real components of the linear susceptibilities, on the other hand, constitute a transition-induced phase mismatch, which in the extreme case of $\left(\Delta \omega_{10}=\right.$ $\left.\Delta \omega_{20}=\Delta \omega\right)$ will limit the coherence length to

$$
l_{c} \equiv \frac{2 \pi}{\beta_{2 \omega}}=\frac{1}{\alpha_{2 \omega}}\left(\frac{\pi}{\Delta \omega}\right)
$$

Thus, even for propagation lengths that are much shorter than the bulk coherence length (as is typical for ISBT's ${ }^{4}$ ), the transition-induced phase mismatch ${ }^{8,9}$ must be considered if the propagation length is comparable with the absorption distance.

Before drawing further conclusions we must consider the limitations of our perturbative, nondepleted derivation in view of the saturable nature of nearresonant SHG. ${ }^{10}$ Since the effects of saturation will become significant at roughly the two-level system's saturation intensity, ${ }^{11}$ the perturbative solution is limited to

$$
\Omega_{\omega}=\Omega_{i j} T_{i j}<1 / 2 \sqrt{T_{i j} / T_{j j}}\left[\left(1+\Delta \omega_{i j}{ }^{2}\right)\right]^{1 / 2},
$$

where $T_{j j}$ is the excited state's lifetime. Near resonance the perturbative treatment is limited to small $\Omega_{\omega}$ 's and hence to small conversion efficiencies, for which we are justified in neglecting the depletion of the first harmonic. Off resonance, large values of $\Omega_{\omega}$ do not lead to saturation, and significant conversion is obtained at distances much shorter than the absorption length $\left(1 / \alpha_{2 \omega}\right)$. Therefore, for large $\Omega_{\omega}$ 's, neglect of the absorption, which is second order in the detuning as is the SHG coefficient, may be a posteriori justified (while the depletion is included). This is typical of SHG in nondispersive, nonlinear crystals but is not relevant to ISBT's, for which the transition frequency to linewidth ratio is limited.

The maximal conversion efficiencies and conversion distances for the perturbative regime and for

(a)
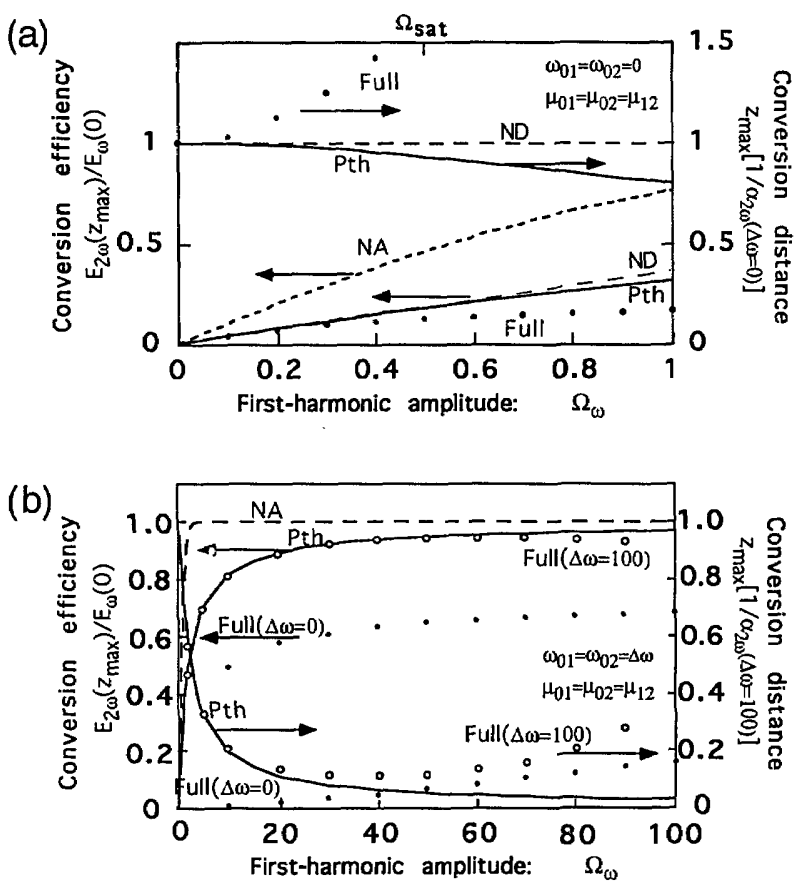

Fig. 1. Second-harmonic amplitude conversion efficiency: $\quad E_{2 \omega}\left(z_{\max }\right) / E_{\omega}(0)$ and conversion length $\left(z_{\max }\right)$ are shown versus normalized amplitudes for (a) moderate and (b) intense fundamental harmonics. The solid curves are the results of the perturbative numerical solutions (Pth), the short-dashed curves are the transparent approximation (NA), and the long-dashed curves (only in the low-intensity case) are the nondepleted approximation (ND). The filled and open circles in (b) are the results of the nonperturbative treatment (Full) for the double-resonant $(\Delta \omega=0)$ and the highly detuned $(\Delta \omega=100)$ cases, respectively. 
(a)

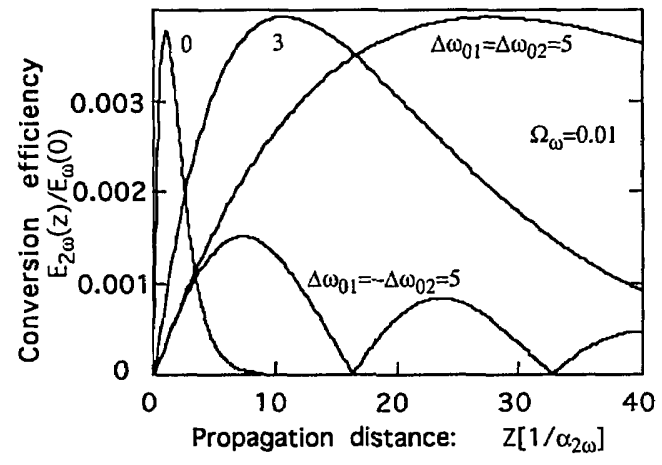

(b)

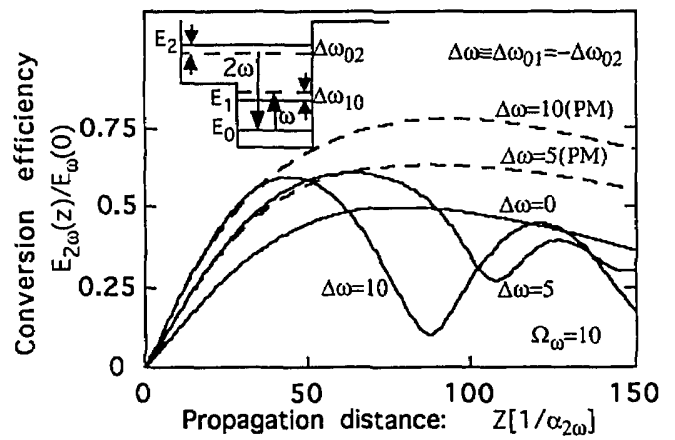

Fig. 2. Numerically derived second-harmonic amplitudes: $E_{2 \omega}(z) / E_{\omega}(0)$ versus normalized propagation lengths for several detunings at (a) a perturbative $\left(\Omega_{\omega}=0.01\right)$ and (b) a saturating $\left(\Omega_{\omega}=10\right)$ normalized intensity. PM, Artificially imposed phase matching.

the large- $\Omega_{\omega}$ regime are plotted versus intensity for the different solutions in Figs. 1(a) and 1(b), respectively. It can be seen from Fig. 1(a) that the perturbative regime breaks down before neglect of the depletion becomes a significant approximation, and therefore the nondepleted solution [Eqs. (4)-(8)] may be used without recourse to numerics. ${ }^{12}$ Figure 1(b) shows that at very large but nonsaturating intensities (highly detuned, $\Delta \omega=100$ ) the conversion length becomes negligible compared with the absorption length-justifying the transparent assumption. Whereas for double resonance the perturbative treatment becomes insufficient at moderate intensities, it breaks down only at very large intensities for the highly detuned case.

Since the treatment of near-resonant fields above the saturation intensity ${ }^{5-7}$ requires a nonperturbative approach, we use a numerical solution of a three-level system. DeTemple et al. ${ }^{7}$ studied general three-wave interactions by reducing the three-level interaction problem to a set of algebraic equations by means of the rotating-wave approximation and using the resultant susceptibilities in a numerical solution of the propagation equations. Boucaud and Julien ${ }^{5}$ and Ikonic et al. ${ }^{6}$ later applied this technique to ISBT's. By considering only the case of SHG we are able to simplify the solution from 15 to 9 equations without further approximation. The numerical solution was used to derive the nonperturbative curves in Fig. 1, showing that perturbation theory breaks down before the nondepleted approximation. Saturation leads to increased conversion lengths and reduced conversion efficiencies but also to a host of novel phenomena that we intend to discuss in detail elsewhere. The numerically obtained second harmonic from a small initial intensity $\left(\Omega_{\omega}=0.01\right)$ and from an intensity well outside the perturbative regime $\left(\Omega_{\omega}=10\right)$ are shown for several detunings in Figs. 2(a) and 2(b), respectively. It can be seen that for this specific case some detuning proves advantageous (although at the expense of greater propagation lengths), contrary to the perturbative conclusions, with the advantage of detuning's becoming more significant when phase matching is externally imposed. The effect of detuning on the conversion efficiency at saturating intensities is in general quite complex and is the subject of further study. Conversion efficiencies approaching unity are still theoretically attainable on resonance, despite absorption, but at much higher intensities and propagation lengths than predicted from the perturbative solution.

In summary, we have used the perturbative solution of SHG, neglecting depletion, to shed light on the mechanisms of resonant SHG and have extended the solution numerically to arbitrary intensities. The situation at saturating intensities becomes much more complex, and optimization of the transitions for SHG should be carried out with respect to the intensities used and the propagation lengths available and with regard to phase-matching issues. The simple form in which we have presented our results should permit the treatment of resonantly enhanced SHG without recourse to unjustified approximations or to numerics. We have shown that double resonance is the optimal conversion condition in the weak-intensity limit, that full conversion is theoretically approachable on resonance, and that the phase mismatch is an inherent consequence of SHG through off-resonant transitions.

\section{References}

1. J. A. Armstrong, N. Bloembergen, J. Ducuing, and P. S. Pershan, Phys. Rev. 127, 1918 (1962).

2. M. M. Fejer, S. J. B. Yoo, R. L. Byer, A. Harwit, and J. S. Harris, Phys. Rev. Lett. 62, 1041 (1989).

3. E. Rosencher, P. Bois, J. Nagle, and S. Delaitre, Electron. Lett. 25, 1063 (1989).

4. Z. Chen, D. Cui, M. Li, C. Jiang, J. Zhou, and G. Yang, Appl. Phys. Lett. 61, 2401 (1992).

5. P. Boucaud and F. H. Julien, J. Phys. III 1, 13 (1991).

6. Z. Ikonic, V. Milanovic, and D. Tjapkin, IEEE J. Quantum Electron. 25, 54 (1989).

7. T. A. DeTemple, L. A. Bahler, and J. Osmundsen, Phys. Rev. A 24, 1950 (1981).

8. G. Almogy, M. Segev, and A. Yariv, Opt. Lett. 19, 1192 (1994).

9. G. Almogy, A. Shakouri, and A. Yariv, Appl. Phys. Lett. 63, 2720 (1993).

10. P. Boucaud, F. H. Julien, D. D. Yang, J. M. Lourtoiz, E. Rosencher, and P. Bois, Opt. Lett. 16, 199 (1991).

11. G. Almogy, M. Segev, and A. Yariv, Phys. Rev. B 48, 10950 (1993).

12. E. Rosencher, J. Appl. Phys. 73, 1909 (1993). 